Generalized symmetric powers and a generalization of the

Kolmogorov-Gel'fand-Buchstaber-Rees theory

Voronov, T. and Khudaverdian, H. M.

2007

MIMS EPrint: 2007.197

Manchester Institute for Mathematical Sciences

School of Mathematics

The University of Manchester

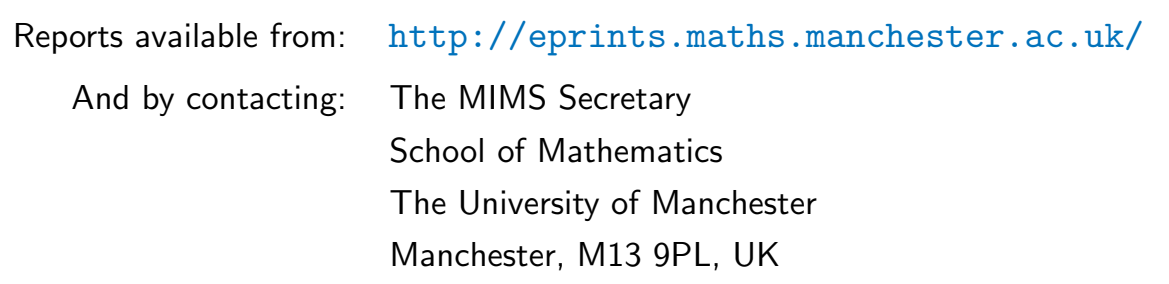

ISSN 1749-9097 


\title{
Generalized symmetric powers and a generalization of the Kolmogorov-Gel'fand-Buchstaber-Rees theory
}

\author{
Th. Th. Voronov [F. F. Voronov] and H. M. Khudaverdian
}

In 1939 Gel'fand and Kolmogorov showed [1] that for a compact Hausdorff topological space $X$, the homomorphisms of the algebra $C(X)$ of continuous functions to the field of real numbers are in a one-to-one correspondence with the points of $X$. Here the algebra $C(X)$ is considered without a topology. This result is less known than its analogue that gave birth to the theory of normed rings. The Gel'fand-Kolmogorov theorem may be viewed as a description of the image of the canonical embedding of $X$ in the infinite-dimensional linear space $V=A^{*}$, where $A=C(X)$, by the system of quadratic equations $\mathbf{f}(1)=1$ and $\mathbf{f}(a)^{2}-\mathbf{f}\left(a^{2}\right)=0$ indexed by elements of $A$. This aspect was recently emphasized by Buchstaber and Rees (see [2] and references therein). They showed that there is a natural embedding in $V$ not only of $X$ itself but also of all its symmetric powers $\operatorname{Sym}^{n} X$. To this end, the algebra homomorphisms should be replaced by the so-called $n$-homomorphisms, and the quadratic equations describing the image of the embedding by certain algebraic equations of higher degree. This theory was motivated by their earlier study of an analogue of Hopf algebras for multivalued groups. Another source is Frobenius' higher group characters.

In this note we give a generalization of the Buchstaber-Rees theory. For a space $X$ we construct a functorial object $\operatorname{Sym}^{p \mid q} X, p, q \geqslant 0$, and for a commutative algebra $A$ with unit we construct a corresponding algebra $S^{p \mid q} A$. We call them 'generalized symmetric powers'. There is a canonical map from $\operatorname{Sym}^{p \mid q} X$ to $V=A^{*}$. To describe its image, we introduce certain algebraic equations, thereby extending the assertions of Gel'fand-Kolmogorov and Buchstaber-Rees. This corresponds to a description of the algebra homomorphisms $S^{p \mid q} A \rightarrow B$ in terms of the new notion of a $p \mid q$-homomorphism. Our work was motivated by results on linear operators on superspaces [3], where our main tool, the 'characteristic function' of a linear map of algebras, comes from. The methods that we propose yield, in particular, a simple direct proof of the main theorem of Buchstaber and Rees.

Let $A$ and $B$ be commutative associative algebras with unit and consider an arbitrary linear map f : $A \rightarrow B$. Its characteristic function is defined to be $R(\mathbf{f}, a, z)=e^{\mathbf{f} \log (1+a z)}$, where $a \in A$ and $z$ is a formal parameter. Example: if $\mathbf{f}$ is an algebra homomorphism, then $R(\mathbf{f}, a, z)=1+\mathbf{f}(a) z$. Algebraic properties of the map $\mathbf{f}$ are reflected in the properties of $R(\mathbf{f}, a, z)$ viewed as a function of the variable $z$. The case when $R(\mathbf{f}, a, z)$ is a polynomial of degree $n$ corresponds to the Buchstaber-Rees theory.

A linear map $\mathbf{f}$ is called a $p \mid q$-homomorphism if $R(\mathbf{f}, a, z)$ is a rational function representable as a ratio of polynomials of degrees $p$ and $q$. Properties of $p \mid q$-homomorphisms follow from general properties of $R(\mathbf{f}, a, z)$. For an arbitrary map $\mathbf{f}, R(\mathbf{f}, a, z)$ has the power series expansion $R(\mathbf{f}, a, z)=1+\psi_{1}(\mathbf{f}, a) z+\psi_{2}(\mathbf{f}, a) z^{2}+\cdots$ at zero, where $\psi_{k}(\mathbf{f}, a)=$ $P_{k}\left(s_{1}, \ldots, s_{k}\right)$. Here $s_{k}=s_{k}(\mathbf{f}, a)=\mathbf{f}\left(a^{k}\right)$ and the $P_{k}$ are the classical Newton polynomials expressing the elementary symmetric functions in terms of sums of powers. The exponential property $R(\mathbf{f}+\mathbf{g}, a, z)=R(\mathbf{f}, a, z) R(\mathbf{g}, a, z)$ holds. Let $R(\mathbf{f}, a, z)$ be defined as a function of $z$ regarded, say, as a complex variable. Let us consider its behaviour at infinity. By a formal transformation we can obtain $R(\mathbf{f}, a, z)=z^{\mathbf{f}(1)} e^{\mathbf{f} \log a} e^{\mathbf{f} \log \left(1+a^{-1} z^{-1}\right)}$

AMS 2000 Mathematics Subject Classification. Primary 13A99, 54C40; Secondary 20C15, 46E25, 46J10, 55R12, 58A50.

DOI 10.1070/RM2007v062n03ABEH004419. 
(cf. [3]). In particular, for $a=1$ we have $R(\mathbf{f}, 1, z)=(1+z)^{\mathbf{f}(1)}$. Assume that $R(\mathbf{f}, a, z)$ does not have an essential singularity. Then $\mathbf{f}(1)=\chi \in \mathbb{Z}$, and the integer $\chi$ is the order of the pole at infinity. We arrive at the expansion $R(\mathbf{f}, a, z)=\sum_{k \leqslant \chi} \psi_{k}^{*}(\mathbf{f}, a) z^{k}$ near infinity, where $\psi_{k}^{*}(\mathbf{f}, a):=e^{\mathbf{f} \log a} \psi_{\chi-k}\left(\mathbf{f}, a^{-1}\right)$. We denote the leading term of the expansion by $e^{\mathbf{f} \log a}=: \operatorname{ber}(\mathbf{f}, a)$ and call it the $\mathbf{f}$-Berezinian of $a \in A$. Note that $a \mapsto \operatorname{ber}(\mathbf{f}, a)$ is in general a partially defined map $A \rightarrow B$. One can see immediately that the f-Berezinian is multiplicative: $\operatorname{ber}\left(\mathbf{f}, a_{1} a_{2}\right)=\operatorname{ber}\left(\mathbf{f}, a_{1}\right) \operatorname{ber}\left(\mathbf{f}, a_{2}\right)$. In the rational case $\operatorname{ber}(\mathbf{f}, a)$ is a ratio of polynomials in the elements $\mathbf{f}\left(a^{k}\right)$.

Here are examples to be kept in mind. If $\mathbf{f}(a)=\operatorname{tr} \boldsymbol{\rho}(a)$ for a matrix representation $\boldsymbol{\rho}: A \rightarrow \operatorname{Mat}(n, B)$, then $R(\mathbf{f}, a, z)=\operatorname{det}(1+\boldsymbol{\rho}(a) z)$ and $\operatorname{ber}(\mathbf{f}, a)=\operatorname{det} \boldsymbol{\rho}(a)$. For a representation by $p|q \times p| q$ matrices, we obtain $R(\mathbf{f}, a, z)=\operatorname{Ber}(1+\boldsymbol{\rho}(a) z)$. In this case $\mathbf{f}(a)=\operatorname{str} \boldsymbol{\rho}(a)$ is the supertrace, and $\operatorname{ber}(\mathbf{f}, a)=\operatorname{Ber} \boldsymbol{\rho}(a)$ is the usual Berezinian.

The Frobenius recursion relations (see [2]) are satisfied by multilinear symmetric functions $\Phi_{k}\left(\mathbf{f}, a_{1}, \ldots, a_{k}\right)$ of elements $a_{i} \in A$ with $\Phi_{k}(\mathbf{f}, a, \ldots, a)=k ! \psi_{k}(\mathbf{f}, a)$. In the case of a matrix representation, $s_{k}(\mathbf{f}, a)=\operatorname{tr} \boldsymbol{\rho}(a)^{k}, \psi_{k}(\mathbf{f}, a)=\operatorname{tr} \Lambda^{k} \boldsymbol{\rho}(a)$, and $\Phi_{k}\left(\mathbf{f}, a_{1}, \ldots, a_{k}\right)=$ $k ! \operatorname{tr}\left(\boldsymbol{\rho}\left(a_{1}\right) \wedge \cdots \wedge \boldsymbol{\rho}\left(a_{k}\right)\right)$.

Going back to the case when $R(\mathbf{f}, a, z)$ is a polynomial in $z$, we can recover the Buchstaber-Rees theory as follows. The degree of $R(\mathbf{f}, a, z)$ in $z$ equals $\mathbf{f}(1)=\chi$, and hence $\chi=n \geqslant 0$. Therefore, $\psi_{k}(\mathbf{f}, a)=0$ for all $k \geqslant n+1$ and all $a \in A$. This is equivalent to the equations $\mathbf{f}(1)=n \in \mathbb{N}$ and $\Phi_{n+1}\left(\mathbf{f}, a_{1}, \ldots, a_{n+1}\right)=0$ for all $a_{i}$, which is exactly the definition of an $n$-homomorphism according to Buchstaber and Rees [2]. In this case $\operatorname{ber}(\mathbf{f}, a)=\psi_{n}(\mathbf{f}, a)$ (in particular, it is a polynomial function of $a$ ), therefore the function $\psi_{n}(\mathbf{f}, a)$ turns out to be multiplicative in $a$, and its polarization $\Phi_{n}\left(\mathbf{f}, a_{1}, \ldots, a_{n}\right) / n$ ! defines an algebra homomorphism $S^{n} A \rightarrow B$. This gives a one-to-one correspondence between the $n$-homomorphisms $A \rightarrow B$ and the algebra homomorphisms $S^{n} A \rightarrow B$.

We define the $p \mid q$-th symmetric power $\operatorname{Sym}^{p \mid q} X$ of a topological space $X$ as the identification space of the Cartesian product $X^{p+q}$ with respect to the action of the group $S_{p} \times S_{q}$ and the relations

$$
\left(x_{1}, \ldots, x_{p-1}, y, x_{p+1}, \ldots, x_{p+q-1}, y\right) \sim\left(x_{1}, \ldots, x_{p-1}, z, x_{p+1}, \ldots, x_{p+q-1}, z\right) .
$$

An algebraic analogue of the space $\operatorname{Sym}^{p \mid q} X$ for a commutative associative algebra $A$ with unit is defined to be the subalgebra $S^{p \mid q} A=\mu^{-1}\left(S^{p-1} A \otimes S^{q-1} A\right)$ of the algebra $S^{p} A \otimes S^{q} A$, where $\mu: S^{p} A \otimes S^{q} A \rightarrow S^{p-1} A \otimes S^{q-1} A \otimes A$ is the multiplication of the last arguments. Example: for $A=\mathbb{C}[x]$, the algebra $S^{p \mid q} A$ will be the algebra of all polynomial invariants of $p|q \times p| q$ matrices (this is a non-trivial statement). There is a relation between the algebra homomorphisms $S^{p \mid q} A \rightarrow B$ and the $p \mid q$-homomorphisms $A \rightarrow B$. To each homomorphism $S^{p \mid q} A \rightarrow B$ the relation assigns canonically a $p \mid q$-homomorphism $A \rightarrow B$. (We have managed to establish the converse result in special cases.) For example, an element $\left[x_{1}, \ldots, x_{p+q}\right] \in \operatorname{Sym}^{p \mid q} X$ defines a $p \mid q$-homomorphism on the algebra $A=$ $C(X): a \mapsto a\left(x_{1}\right)+\cdots+a\left(x_{p}\right)-\cdots-a\left(x_{p+q}\right)$. In general, an integral linear combination of algebra homomorphisms of the form $\sum n_{\alpha} \mathbf{f}_{\alpha}$, where $n_{\alpha} \in \mathbb{Z}$, is a $p \mid q$-homomorphism with $p=\sum_{n_{\alpha}>0} n_{\alpha}, q=-\sum_{n_{\alpha}<0} n_{\alpha}$, and $\chi=\sum n_{\alpha}$.

The condition that $\mathbf{f}: A \rightarrow B$ is a $p \mid q$-homomorphism can be expressed by the equations $\mathbf{f}(1)=p-q$ and $\left|\psi_{k}(\mathbf{f}, a), \ldots, \psi_{k+q}(\mathbf{f}, a)\right|_{q+1}=0$ for $k \geqslant p-q+1$ (a Hankel determinant; see [3]). This system of polynomial equations for the 'coordinates' of the linear map $\mathbf{f}$ should describe, in particular, the image of $\operatorname{Sym}^{p \mid q} X$.

Our results may have an application to topological ramified coverings (cf. [4]). We thank V. M. Buchstaber for a fruitful discussion. 


\section{Bibliography}

[1] И. М. Гельфанд, А. Н. Колмогоров, Докл. АН СССР 22 (1939), 11-15; English transl., I. M. Gel'fand and A. N. Kolmogorov, Selected works of A. N. Kolmogorov, vol. I, Kluwer, Dordrecht 1991, pp. 291-297.

[2] В. М. Бухштабер, Э. Г. Рис, УМН 59:1 (2004), 125-144; English transl., V. M. Buchstaber and E. G. Rees, Russian Math. Surveys 59:1 (2004), 125-145.

[3] H. M. Khudaverdian and Th. Th. Voronov, Lett. Math. Phys. 74:2 (2005), 201-228.

[4] V.M. Buchstaber and E. G. Rees, "Frobenius $n$-homomorphisms, transfers and branched coverings", Math. Proc. Cambridge Philos. Soc. (to appear); arXiv: math.RA/0608120.

Th. Th. Voronov [F. F. Voronov]

School of Mathematics, University of Manchester, United Kingdom

E-mail: theodore.voronov@manchester.ac.uk

\section{H. M. Khudaverdian}

School of Mathematics, University of Manchester, United Kingdom

E-mail: khudian@manchester.ac.uk
Presented by V. M. Buchstaber Received 08/JAN/07 Translated by THE AUTHORS 